Regular Article

pISSN: 2287-2396, eISSN: 2287-240X

Journal of Forest Science

Vol. 30, No. 1, pp. 1-14, February, 2014

http://dx.doi.org/10.7747/JFS.2014.30.1.1

\title{
Assessment of Timber Harvest in Tropical Rainforest Ecosystem of South West Nigeria and Its Implication on Carbon Sequestration
}

Victor A. Adekunle, Amadu Lawal and Adewole O. Olagoke*

Department of Forestry and Wood Technology, Federal University of Technology, P.M.B. 704, Akure 340001, Nigeria

\begin{abstract}
Timber harvest in natural forests and its implications on carbon sequestration were investigated in the Southwestern Nigeria. Data on timber harvest from forest estates for a 3-year period were collected from the official record of States' Forestry Department. The data registered the species, volume and number of timbers exploited during the study period. The data were analyzed accordingly for rate of timber harvest and carbon value of the exploited timbers using existing biomass functions. Values were compared for significant differences among states using one way analysis of variance. The results showed that the most exploited logs, in terms of volume and number of trees, have the highest amount of carbon removal. There was a variation in type of timber species being exploited from each state. The total number of harvested trees from Oyo, Ondo, Ogun, Ekiti and Osun were estimated at 100,205; 111,789; 753; 15,884 and 18,153 respectively. Total quantity of carbon removed for the 3-year period stood at 2.3 million metric tons, and this translated to 8.4 million metric tons of $\mathrm{CO}_{2}$. The annual carbon and $\mathrm{CO}_{2}$ removal therefore were estimated at $760,120.73$ tons and 2.8 million tons/ year respectively. There were significant differences $(\mathrm{p}<0.05)$ in the amount of $\mathrm{CO}_{2}$ removed from the five states. Based on our result, we inferred that there is increasing pressure on economic tree species and it is plausible that they are becoming scarce from the forests in Southwestern Nigeria.. If the present rate of log removal is not controlled, forests could become carbon source rather than carbon sink and the on biological conservation, wood availability and climate change may turn out grave. For the forest to perform its environmental role as carbon sink, urgent conservation measures and logging policies are needed to be put in place.
\end{abstract}

Key Words: Logging, Timber harvest, Biomass removal, Carbon emission

\section{Introduction}

Climate change is posing a dreadful threat to the development and poverty reduction processes in the poorest and most vulnerable regions of the world today. It is considered as one of the most serious threats to sustainable development, with its adverse impacts expected on the environment, human health, food security, economic activity, natural resources and physical infrastructure (IISD 2009;
Wheeler and von Braun 2013). Changes in climatic conditions have significant impacts on the capacity of the forest to provide vital ecosystem services and the well-being of people in African countries where there are low adaptation strategies and mitigation options are the most affected. The low adaptation capacity is not unconnected with the extreme poverty, frequent natural disasters such as droughts and floods and an agricultural system that heavily dependent on rainfall (Huq et al. 2003). For instance, the greatest pro-

Received: December 3, 2012. Revised: November 25, 2013. Accepted: November 25, 2013.

Corresponding author: Adewole O. Olagoke

Department of Forestry and Wood Technology, Federal University of Technology, P.M.B. 704, Akure 340001, Nigeria

Tel: 2348102919537, Fax: 2348102919537, E-mail: adewoleolagoke@gmail.com 
portion of Nigerian population lives in rural areas, and they depend solely on renewable natural resources for survival. The daily activities of this set of people in the forest are culpable of leading to forest degradation and alteration in environmental conditions. Houghton (1991) remarked that the estimates of carbon fluxes from deforestation, land cover change and other disturbances depend on the knowledge of forest carbon stocks prior to the disturbance.

Logging has to do with the harvesting of tree species, and consequently the removal of merchantable aboveground wood biomass that acts as carbon sink from the forest for sawn wood production. Above-ground biomass consists of all living biomass above the soil such as stem, stump, branches, bark, seeds, and foliage while below-ground biomass consists of all living roots excluding fine roots (less than $2 \mathrm{~mm}$ in diameter) (Drake et al. 2003; Samalca et al. 2007). Therefore, estimates of the total above-ground biomass in forest ecosystems are critical for carbon dynamics studies, as plant biomass is an important indicator in carbon sequestration. The amount of carbon sequestered can be inferred from the biomass change since $50 \%$ of the forest dry biomass is approximately carbon (Losi et al. 2003; Montagu et al. 2005). For this purpose, one needs to know how much biomass is lost or accumulated over time. These estimates provide initial conditions for ecosystem and biogeochemical models that simulate the exchange of carbon and energy between the atmosphere and biosphere (Potter 1999). In addition, biomass estimation is very useful in evaluating the amount of carbon sequestrated in the afforestation and reforestation Clean Development Mechanism (AR-CDM) project under the Kyoto Protocol (Hiratsuka et al. 2004). There are two major methods of biomass estimation (the destructive and non-destructive). The destructive method requires felling of trees but the non-destructive method does not require the trees to be felled (Montes et al. 2000). For non-destructive method, tree growth variables are obtained for computing the total volume $\left(\mathrm{m}^{3}\right)$. Wood density of tree species, which are available from literature, proved valuable in converting the measured volume into biomass estimate (Feamside 1997; Aboal et al. 2005). Such estimate is used to quantify the amount of carbon removal from the ecosystem from the general knowledge that carbon is always $50 \%$ of biomass estimate (Samalca 2007).

Many biomass and carbon estimation studies conducted focused on aboveground forest biomass (Aboal et al. 2005; Brown 1997; Kraenzel et al. 2003; Laclau 2003; Losi et al. 2003) because it accounts for the majority of the total accumulated biomass in the forest ecosystem. Aboveground tree biomass in turn is estimated using allometric equations (Hiratsuka et al. 2006). However, where allometric equations are not available or feasible, Birdsey (1996) reported that timber cruise estimates of merchantable volume could be converted to biomass and weight by multiplying by a conversion factor for the corresponding region and forest type to determine the carbon mass in living standing trees. Renasingbe and Abasari (2008) also estimated tree biomass, weight and carbon storage from volumes using the same conversion factor. In view of the above, this study focuses on the assessment of above-ground forest biomass from volumes of trees exploited between 2003 and 2005 to estimate the amount of carbon removed during the study period.

\section{Methodology}

\section{The study area}

This study was carried out in Southwest (SW) Nigeria. It is one of the six geographical zones of country. The zone lies between longitude $2^{\circ} 31^{\prime}$ and $6^{\circ} 00^{\prime}$ East and Latitude $6^{\circ} 21^{\prime}$ and $8^{\circ} 37^{\prime} \mathrm{N}$ (Adekunle and Olagoke 2010) with a total land area of $77,818 \mathrm{~km}^{2}$ and a projected population of 28, 767, 752 in 2002 (NPC 1991). SW Nigeria is bounded in the East by Edo and Delta states, in the North by Kwara and Kogi states, in the West by the Republic of Benin and in the south by the Gulf of Guinea. The region is made up of six states, out of the 36 states in the country, and is majorly inhabited by the Yorubas, one of the major tribes in the country. The climate of South West Nigeria is tropical in nature and it is characterized by wet and dry seasons. The temperature ranges between $21^{\circ} \mathrm{C}$ and $34^{\circ} \mathrm{C}$ while the annual rainfall ranges between $1500 \mathrm{~mm}$ and $3000 \mathrm{~mm}$. The wet season is associated with the South West monsoon wind from the Atlantic Ocean while the dry season is associated with the North East trade wind from the Sahara desert. Most of the soils in the study area developed from undifferentiated igneous and metamorphic, pre-cambrian basement complex rocks such as granite, biotite, gneiss, biotite schist, quartz schist and quartzite (Adesodun et al. 
2007). The vegetation of South West Nigeria is made up of fresh water swamp and mangrove forest at the belt, the low land in forest stretches inland to Ogun and part of Ondo state while the secondary forest is towards the northern boundary where derived and southern Savannah exists.

\section{Method of data collection}

Secondary data for the reserved and unreserved forests (free areas) were used for this study. Forest reserves are portion of forest estates constituted by law and gazette by state or local government. All other woodlands that are not under reservation are referred to as 'free areas' in this study. There are about 1,600 forest reserves in Nigeria that are disproportionately scattered across the states, while the free areas are in form of woodlots, trees on farmlands, private plantations and farm forestry. Entrance and activities in reserves are restricted and controlled by the government but government's control on the free area is limited to the collection of logging fees. Data on the volume $\left(\mathrm{m}^{3}\right)$, timber species and number of logs that were exploited, on monthly basis, in three year period (2008 and 2010) were collected from five randomly selected States out of the six States in the zone. The selected States are Ondo, Osun, Oyo, Ekiti and Ogun States. These are states with active logging activities due to the abundance of timber resources in their respective reserves. The Department of Forestry offices located in the State Capital of each of the selected States were visited for data collection. The logging data were collected from official records, files and reports in each state. To gather some additional information on each reserve, the list and management plan of all the reserves in each state were obtained.

\section{Data analysis}

Estimation of the Above-ground biomass was carried out by multiplying the volume of each tree with its respective wood density as also done by Feamside (1997). The densities were obtained from literatures which include the Nigerian Standard Code of Practice (1973) and Dinwoodie (1981). The carbon content was estimated as $50 \%$ of the dry weight or biomass (MacDicke 1997; Penman 2003; and IPCC 2006). Estimate of annual carbon storage in metric tons was estimated by dividing the total carbon for the three years by three. The relationship be- tween carbon content and carbon dioxide was used to convert carbon in the wood to $\mathrm{CO}_{2}$. This represents the amount of carbon the wood could sequestrate if they have not been logged or the amount of $\mathrm{CO}_{2}$ to be removed into the atmosphere if they act as carbon source. The relationship is given as follows:

$1 \mathrm{tC}=3.67 \mathrm{t} \mathrm{CO}_{2}($ NIACS 2008; Renasingbe and Abasari 2008).

The Carbon and $\mathrm{CO}_{2}$ contents of the individual trees were estimated in metric ton $(\mathrm{t})$. Data collected were analyzed separately for each state and then one way Analysis of Variance (ANOVA) was used to compare and test for significant difference in the estimates. Natural Logarithmic values of all the variables for the fifteen most exploited tree species were used for the comparison. The states' variables that we compared were the number of trees, cubic volume and the amount of carbon. The analyses were performed using SPSS for windows 17.0 version.

\section{Results}

The list of reserves in each of the selected states is presented in table 1. As presented in the Table, Ogun State has the largest area of gazetted forest estate under reservation (266,924 ha) in Southwest Nigeria. This is followed by Oyo State $(114,441.40 \mathrm{ha})$. The area of reserved forest in Osun State is about $58,893.32$ ha, and the least forest estate was located in Ondo State (3,075.66 ha) which has the highest number of reserves (16). The botanical names of all the species exploited during the 3-year period from South West Nigeria, their individual families, their respective volumes, density, biomass, carbon stock and $\mathrm{CO}_{2}$ equivalent are presented in Table 2. Generally, Ceiba pentandra $(16,875)$ in Bombacaceae family was the most exploited species in South West Nigeria. While Ficus exasperata $(10,402)$, Milletia thonningii $(9,261)$ and Mitragyna stipulosa $(8,362)$ were next to Ceiba pentandra in the level of exploitation, only $7.56 \%$ species recorded one number of exploited tree during the study period. Also, Ceiba pentandra $\left(640,021.04 \mathrm{~m}^{3}\right)$ recorded the highest volume of extraction. This is followed by Funtumia elastica $\left(414,449.50 \mathrm{~m}^{3}\right)$ and Anogeissus leiocarpa $\left(387,606.24 \mathrm{~m}^{3}\right)$. The least volume extraction $\left(0.91 \mathrm{~m}^{3}\right)$ was 
Table 1. List of forest reserves and their respective sizes in the five States

\begin{tabular}{|c|c|c|c|c|c|c|c|c|c|}
\hline \multicolumn{2}{|l|}{ Oyo state } & \multicolumn{2}{|l|}{ Ondo state } & \multicolumn{2}{|c|}{ Ogun state } & \multicolumn{2}{|c|}{ Osun state } & \multicolumn{2}{|l|}{ Ekiti state } \\
\hline Forest reserves & $\begin{array}{l}\text { Size } \\
\text { (ha) }\end{array}$ & Forest reserves & $\begin{array}{l}\text { Size } \\
\text { (ha) }\end{array}$ & $\begin{array}{l}\text { Forest } \\
\text { reserves }\end{array}$ & $\begin{array}{l}\text { Size } \\
\text { (ha) }\end{array}$ & $\begin{array}{c}\text { Forest } \\
\text { reserves }\end{array}$ & $\begin{array}{l}\text { Size } \\
\text { (ha) }\end{array}$ & $\begin{array}{c}\text { Forest } \\
\text { reserves }\end{array}$ & $\begin{array}{l}\text { Size } \\
\text { (ha) }\end{array}$ \\
\hline Gambari & 11,431 & Akure & 6,993 & Area J3, J4, J6 & 130,550 & Ago Owu & 32,116 & Ogbese & 7,252 \\
\hline Ijaiye & 28,491 & Akure-Ofosu Ext. & 2,089 & Olokemeji & 5,888 & Oba hill & 3,367 & Egbe & 1,022 \\
\hline Osho & 3,704 & Akure-Ofosu & 40,145 & Arakanga & 239 & Ila & 259 & Eda I \& II & 906 \\
\hline Olokemeji & 7,511 & $\mathrm{Ala}$ & 19,943 & Ilaro & 4,608 & Ife North & 8,598 & Ogotun group & 1,569 \\
\hline Lanlate & 7,507 & Eba Island & 1,813 & Edun stream & 97 & Oni & 283 & Aramoko & 1,966 \\
\hline Igangan & 39,627 & Idanre (OA5) & 54,053 & Eggua & 4,147 & Ikeji/Ipetu & 3,548 & Ikere & 1,419 \\
\hline Olaseinde & 686 & Ifon & 28,231 & Aworo & 21,299 & & & Little Ose & 2,662 \\
\hline Olla Hills (Okoo-Iroo) & 2,300 & Ipele-Idoani & 4,144 & Imeko & 95,488 & & & Ise & 5,677 \\
\hline Opara & 3,544 & Irele (OA11) & 3,600 & Ohunbe & 4,608 & & & Eporo & 4,662 \\
\hline \multirow[t]{7}{*}{ Baasi } & 1,144 & Ojigbogbini & 2,809 & & & & & Isan/ Ayede & 2,585 \\
\hline & & Okeluse & 11,137 & & & & & & \\
\hline & & $\begin{array}{c}\text { Oluwa (OA1, OA2, } \\
\text { OA3, OA6) }\end{array}$ & 87,816 & & & & & & \\
\hline & & Onisere (OA4) & 9,842 & & & & & & \\
\hline & & Otu (OA4) & 8,490 & & & & & & \\
\hline & & Owo & 24,216 & & & & & & \\
\hline & & Oyinmo & 2,245 & & & & & & \\
\hline Total & $114,441.40$ & 307 & 566.00 & & $66,924.00$ & & $8,839.32$ & \multicolumn{2}{|c|}{$29,720.00$} \\
\hline
\end{tabular}

recorded for about $2.91 \%$ species in the study area. In term of biomass, carbon stock and $\mathrm{CO}_{2}$ removed, anogeissus leicocarpa recorded the highest values, and Cassia siamea, Butyrospermum paradoxum and Bridelia artroviridis were noted for the least values of biomass removal.

In Oyo State, Anogeissus leicocarpus (6,590) of the family Combretaceae was the most exploited species but, Mansonia altisima (62) of the family Sterculiaceae was the least exploited species. In Ondo State, Mitragyna stipulosa $(8,353)$ of the family Rubiaceae was the most exploited while Blighia sapida (47) of the family Sapindaceae was the least exploited species. The most exploited species in Ogun, Osun and Ekiti States was Ceiba pentandra in Bombacaceae family with 30 trees in Ogun State, 2426 in Osun State and 1584 in Ekiti State. However, $11.5 \%$ of the tree species in Osun State, $18.9 \%$ of those in Ogun Sate and $6.67 \%$ in Ekiti State had only a tree that was logged during the three year period. For tree volume estimate, the highest annual volume was exploited in Oyo State $\left(1,910,348.33 \mathrm{~m}^{3}\right)$. This is followed by Osun State with an annual volume removal of $713,025.00 \mathrm{~m}^{3}$ and the least, 9,087.03 $\mathrm{m}^{3}$ in Ogun State. The annual volume of wood removed from Ondo and Ekiti
State were estimated at $98,414.87 \mathrm{~m}^{3}$ and $44,875 \mathrm{~m}^{3}$ respectively. For the diversity of logged trees, 57 species, belonging to 29 families, were exploited in Oyo State; 61 species belonging to 24 families were exploited in Ondo State; 74 species in 33 families were removed from Ogun State, 60 species belonging to 25 families were removed from Ekiti State and 130 species in 42 families were exploited in Osun State. Osun was the state with the highest species diversity of the felled logs.

In Oyo State, the highest carbon released was from Anogeissus leicocarpus (442,193.85 tons). This is followed by Cassia siamea (434,001.26 tons) and the least carbon released was from Cordia millenii (2495.31). Terminalia super$b a$ and Afzelia africana recorded the highest carbon release in Ondo State and the estimated values are 31,932.84 and $31,677.39$ metric tons respectively. However, Borassus aethiopica (4.93 tons) had the least estimated carbon value. In Ogun State, Albizia zygia was the most exploited species and as a result, it has the highest amount of $\mathrm{CO}_{2}(2,317.27$ tons). Next to Albizia zygia in term of carbon release is Milicia excelsa (2,307.40 tons) while Cedrella odorata which was the least exploited species also has the least amount of 
Table 2. List of exploited tree species, families, and quantity of carbon and $\mathrm{CO}_{2}$ released for a 3-year period in southwest Nigeria

\begin{tabular}{|c|c|c|c|c|c|c|c|c|}
\hline $\mathrm{S} / \mathrm{N}$ & Family & Tree Species & $\begin{array}{l}\text { Number } \\
\text { of tree }\end{array}$ & Volume $\left(\mathrm{m}^{3}\right)$ & $\begin{array}{l}\text { Density } \\
\left(\mathrm{kg} / \mathrm{m}^{3}\right)\end{array}$ & $\begin{array}{l}\text { Biomass } \\
\text { (tons) }\end{array}$ & $\begin{array}{l}\text { Carbon stock } \\
\quad \text { (tons) }\end{array}$ & $\mathrm{CO}_{2}$ (tons) \\
\hline 1 & Anacardiaceae & Lannea acida & 36 & 2719.776 & 540 & 1468.68 & 734.34 & 2695.03 \\
\hline 2 & " & Lannea barteri & 151 & 17793.2172 & 540 & 9608.34 & 4804.17 & 17631.30 \\
\hline 3 & $"$ & Lannea welwitschii & 1376 & 42109.609 & 640 & 26950.15 & 13475.07 & 49453.52 \\
\hline 4 & $"$ & Mangiferia indica & 956 & 55107.7902 & 640 & 35268.99 & 17634.49 & 64718.59 \\
\hline 5 & " & Pseudospondias microcarpa & 3 & 353.5077 & 695 & 245.69 & 122.84 & 450.84 \\
\hline 6 & $"$ & Spondias mombin & 403 & 26546.466 & 464 & 12317.56 & 6158.78 & 22602.72 \\
\hline 7 & Annonaceae & Cleistopholis libericus & 16 & 304.36 & 410 & 124.79 & 62.39 & 228.99 \\
\hline 8 & $"$ & Cleistopholis patens & 872 & 53562.775 & 410 & 21960.74 & 10980.37 & 40297.95 \\
\hline 9 & Apocynaceae & Alstonia boonei & 1014 & 8975.4154 & 430 & 3859.43 & 1929.71 & 7082.05 \\
\hline 10 & " & Alstonia congonesis & 1412 & 84924.38 & 432 & 36687.33 & 18343.67 & 67321.25 \\
\hline 11 & $"$ & Funtumia elastica & 6961 & 414449.504 & 450 & 186502.28 & 93251.14 & 342231.68 \\
\hline 12 & $"$ & Holarrhena floribunda & 18 & 318.6 & 540 & 172.04 & 86.02 & 315.70 \\
\hline 13 & Arecaceae & Hyphaena thebaica & 3 & 353.5077 & 640 & 226.24 & 113.12 & 415.16 \\
\hline 14 & Bignoniaceae & Nerwbouldia laevis & 1 & 0.91 & 540 & 0.49 & 0.25 & 0.90 \\
\hline 15 & " & Spathodea campamulata & 5 & 589.1795 & 340 & 200.32 & 100.16 & 367.59 \\
\hline 16 & Bombacaceae & Bombax buonopozense & 827 & 11127.6981 & 330 & 3672.14 & 1836.07 & 6738.38 \\
\hline 17 & " & Ceiba pentandra & 16875 & 640021.04 & 260 & 166405.47 & 83202.74 & 305354.04 \\
\hline 18 & Boraginaceae & Cordia millenii & 955 & 36541.2559 & 340 & 12424.03 & 6212.01 & 22798.09 \\
\hline 19 & " & Cordia platythyrsa & 2 & 5.85 & 340 & 1.99 & 0.99 & 3.65 \\
\hline 20 & " & Corynanthe pachyceras & 2183 & 49.5 & 640 & 31.68 & 15.84 & 58.13 \\
\hline 21 & " & Crossopteryx febrifuga & 1 & 117.8359 & 640 & 75.41 & 37.71 & 138.39 \\
\hline 22 & " & Cylicodiscus gabunensis & 7 & 824.8513 & 640 & 527.90 & 263.95 & 968.71 \\
\hline 23 & Burseraceae & Canarium africana & 6 & 2.72 & 450 & 1.22 & 0.61 & 2.25 \\
\hline 24 & " & Canarium schweinfurthii & 588 & 10209.3997 & 479 & 4890.30 & 2445.15 & 8973.71 \\
\hline 25 & " & Carapa procerra & 593 & 386.2877 & 550 & 212.46 & 106.23 & 389.86 \\
\hline 26 & " & Cassia nodosa & 8 & 5.45 & 630 & 3.43 & 1.72 & 6.30 \\
\hline 27 & " & Cassia siamea & 5307 & 303221.73 & 780 & 236512.95 & 118256.47 & 434001.26 \\
\hline 28 & " & Cassia sieberiana & 389 & 45838.165 & 640 & 29336.43 & 14668.21 & 53832.34 \\
\hline 29 & Caesalpiniaceae & Combretodendron macrocarpum & 250 & 1545.511 & 460 & 710.94 & 355.47 & 1304.57 \\
\hline 30 & " & Erythrophleum africanum & 10 & 46.08 & 600 & 27.65 & 13.82 & 50.73 \\
\hline 31 & " & Erythrophleum ivorensis & 2288 & 132609.283 & 832 & 110330.92 & 55165.46 & 202457.24 \\
\hline 32 & Caesalpinioideae & Daniellia ogea & 6442 & 341500.77 & 550 & 187825.42 & 93912.71 & 344659.65 \\
\hline 33 & " & Daniellia oliveri & 499 & 31115.01 & 550 & 17113.26 & 8556.63 & 31402.82 \\
\hline 34 & " & Delonix regia & 86 & 4913.7119 & 485 & 2383.15 & 1191.58 & 4373.08 \\
\hline 35 & " & Detarium microcapum & 5563 & 317848.6 & 640 & 203423.10 & 101711.55 & 373281.40 \\
\hline 36 & " & Dialium guineesis & 5536 & 313035.535 & 640 & 200342.74 & 100171.37 & 367628.93 \\
\hline 37 & $"$ & Hylondendron gabunense & 359 & 41378.29 & 640 & 26482.11 & 13241.05 & 48594.66 \\
\hline 38 & Chrysobalanaceae & Parinari congensis & 1 & 0.91 & 540 & 0.49 & 0.25 & 0.90 \\
\hline 39 & " & Parinari excelsa & 1 & 117.8359 & 540 & 63.63 & 31.82 & 116.76 \\
\hline 40 & Combretaceae & Anogeissus leiocarpa & 6685 & 387606.24 & 720 & 279076.49 & 139538.25 & 512105.36 \\
\hline 41 & $"$ & Terminalia ivorensis & 3013 & 55812.551 & 625 & 34882.84 & 17441.42 & 64010.02 \\
\hline 42 & " & Terminalia superba & 3518 & 106576.16 & 540 & 57551.13 & 28775.56 & 105606.32 \\
\hline 43 & Connaraceae & Byrsocarpus coccineus & 2 & 235.6718 & 720 & 169.68 & 84.84 & 311.37 \\
\hline 44 & Cucurbitaceae & Momordica cabraei & 93 & 10958.74 & 640 & 7013.59 & 3506.80 & 12869.94 \\
\hline 45 & Ebenaceae & Diospyros canaliculata & 2 & 235.6718 & 720 & 169.68 & 84.84 & 311.37 \\
\hline 46 & " & Diospyros monbuttensis & 103 & 5778.6582 & 864 & 4992.76 & 2496.38 & 9161.72 \\
\hline 47 & " & Drypetes paxii & 1 & 117.8359 & 700 & 82.49 & 41.24 & 151.36 \\
\hline
\end{tabular}


Table 2. Continued

\begin{tabular}{|c|c|c|c|c|c|c|c|c|}
\hline $\mathrm{S} / \mathrm{N}$ & Family & Tree Species & $\begin{array}{l}\text { Number } \\
\text { of tree }\end{array}$ & Volume $\left(\mathrm{m}^{3}\right)$ & $\begin{array}{l}\text { Density } \\
\left(\mathrm{kg} / \mathrm{m}^{3}\right)\end{array}$ & $\begin{array}{l}\text { Biomass } \\
\text { (tons) }\end{array}$ & $\begin{array}{l}\text { Carbon stock } \\
\text { (tons) }\end{array}$ & $\mathrm{CO}_{2}$ (tons) \\
\hline 48 & Euphorbiaceae & Bridelia artroviridis & 1 & 0.91 & 470 & 0.43 & 0.21 & 0.78 \\
\hline 49 & $"$ & Bridelia ferruginea & 440 & 25139.921 & 470 & 11815.76 & 5907.88 & 21681.92 \\
\hline 50 & $"$ & Bridelia micrantha & 1 & 117.8359 & 470 & 55.38 & 27.69 & 101.63 \\
\hline 51 & $"$ & Butyrospermum paradoxum & 5438 & 310767.2759 & 720 & 223752.44 & 111876.22 & 410585.72 \\
\hline 52 & $"$ & Hevea brasiliensis & 4 & 471.3436 & 660 & 311.09 & 155.54 & 570.84 \\
\hline 53 & $"$ & Macaranga barteri & 3 & 353.5077 & 640 & 226.24 & 113.12 & 415.16 \\
\hline 54 & $"$ & Phyllanthus angolensis & 1 & 0.91 & 544 & 0.50 & 0.25 & 0.91 \\
\hline 55 & $"$ & Phyllanthus discoideus & 1035 & 363.3 & 760 & 276.11 & 138.05 & 506.66 \\
\hline 56 & $"$ & Ricinodendrum heudelotti & 4013 & 241006.1 & 200 & 48201.22 & 24100.61 & 88449.24 \\
\hline 57 & Fabaceae & Afrormosia elata & 4756 & 145.2 & 640 & 92.93 & 46.46 & 170.52 \\
\hline 58 & $"$ & Afzelia africana & 3512 & 196105.108 & 780 & 152961.98 & 76480.99 & 280685.24 \\
\hline 59 & $"$ & Albizia zygia & 27 & 2686.85 & 470 & 1262.82 & 631.41 & 2317.27 \\
\hline 60 & $"$ & Albizia ferruginea & 59 & 6952.318 & 550 & 3823.77 & 1911.89 & 7016.63 \\
\hline 61 & $"$ & Albizia lebbeck & 3495 & 94165.68 & 590 & 55557.75 & 27778.88 & 101948.47 \\
\hline 62 & $"$ & Albizia zygia & 5440 & 292533.7 & 470 & 137490.84 & 68745.42 & 252295.69 \\
\hline 63 & $"$ & Gossweilerodendron balsamiferon & 104 & 6.9 & 640 & 4.42 & 2.21 & 8.10 \\
\hline 64 & $"$ & Milletia griffoniana & 2 & 235.6718 & 640 & 150.83 & 75.41 & 276.77 \\
\hline 65 & $"$ & Milletia thonningii & 9261 & 190316.675 & 632 & 120280.14 & 60140.07 & 220714.05 \\
\hline 66 & $"$ & Parkia biglobosa & 56 & 5661.123 & 540 & 3057.01 & 1528.50 & 5609.61 \\
\hline 67 & $"$ & Parkia clappertoniana & 5280 & 301679.05 & 440 & 132738.78 & 66369.39 & 243575.66 \\
\hline 68 & $"$ & Pterocarpus erinaceus & 10 & 1178.359 & 640 & 754.15 & 377.07 & 1383.86 \\
\hline 69 & $"$ & Pterocarpus osun & 446 & 26089.736 & 640 & 16697.43 & 8348.72 & 30639.79 \\
\hline 70 & $"$ & Pterocarpus santalinoides & 4904 & 1696.1672 & 640 & 1085.55 & 542.77 & 1991.98 \\
\hline 71 & $"$ & Pycnanthus angolensis & 1845 & 55920.018 & 544 & 30420.49 & 15210.24 & 55821.60 \\
\hline 72 & Flacourtiaceae & Scottellia coriacea & 45 & 5302.615 & 640 & 3393.67 & 1696.84 & 6227.39 \\
\hline 73 & Glusiaceae. & Allanblankia floribunda & 2 & 0.91 & 860 & 0.78 & 0.39 & 1.44 \\
\hline 74 & Guttiferae & Garcinia smeathmannii & 1 & 117.8359 & 640 & 75.41 & 37.71 & 138.39 \\
\hline 75 & $"$ & Symphonia globulifa & 18 & 599.18 & 470 & 281.61 & 140.81 & 516.76 \\
\hline 76 & Humiriaceae & Sacoglottis gabonensis & 2 & 1.82 & 540 & 0.98 & 0.49 & 1.80 \\
\hline 77 & $"$ & Irvingia gabonensis & 19 & 1142.6613 & 900 & 1028.40 & 514.20 & 1887.11 \\
\hline 78 & $"$ & Irvingia wombulu & 2948 & 19089.386 & 900 & 17180.45 & 8590.22 & 31526.12 \\
\hline 79 & Leguminosae & Brachystegia eurycoma & 4877 & 66030.199 & 600 & 39618.12 & 19809.06 & 72699.25 \\
\hline 80 & $"$ & Distemonanthus benthamianus & 1434 & 4558.3112 & 640 & 2917.32 & 1458.66 & 5353.28 \\
\hline 81 & " & Erythrina senegalensis & 10 & 1178.359 & 640 & 754.15 & 377.07 & 1383.86 \\
\hline 82 & Loganiaceae & Anthocleista procera & 20 & 2356.718 & 640 & 1508.30 & 754.15 & 2767.73 \\
\hline 83 & $"$ & Anthocleista schweinfurthii & 3142 & 179521.89 & 640 & 114894.01 & 57447.00 & 210830.51 \\
\hline 84 & " & Anthostema aubreyanum & 6 & 707.0154 & 640 & 452.49 & 226.24 & 830.32 \\
\hline 85 & Malvaceae & Cola lateritia & 6 & 122.3959 & 640 & 78.33 & 39.17 & 143.74 \\
\hline 86 & " & Cola gigantia & 3172 & 202387.59 & 460 & 93098.29 & 46549.15 & 170835.36 \\
\hline 87 & $"$ & Cola nitida & 141 & 16614.86 & 640 & 10633.51 & 5316.76 & 19512.49 \\
\hline 88 & $"$ & Hildegardia barteri & 4 & 471.3436 & 640 & 301.66 & 150.83 & 553.55 \\
\hline 89 & Meliaceae & Azadiracta indica & 93 & 5257.439 & 840 & 4416.25 & 2208.12 & 8103.82 \\
\hline 90 & $"$ & Entandrophragma cylindricum & 541 & 17657.6515 & 592 & 10453.33 & 5226.66 & 19181.86 \\
\hline 91 & $"$ & Entandrophragma utile & 3199 & 3828.0895 & 832 & 3184.97 & 1592.49 & 5844.42 \\
\hline 92 & $"$ & Guarea cedrata & 7573 & 198.57 & 635 & 126.09 & 63.05 & 231.38 \\
\hline 93 & " & Guarea thomposonii & 10 & 1178.359 & 635 & 748.26 & 374.13 & 1373.05 \\
\hline 94 & $"$ & Khaya grandifoliola & 767 & 6136.809 & 720 & 4418.50 & 2209.25 & 8107.95 \\
\hline
\end{tabular}


Table 2. Continued

\begin{tabular}{|c|c|c|c|c|c|c|c|c|}
\hline $\mathrm{S} / \mathrm{N}$ & Family & Tree Species & $\begin{array}{c}\text { Number } \\
\text { of tree }\end{array}$ & Volume $\left(\mathrm{m}^{3}\right)$ & $\begin{array}{l}\text { Density } \\
\left(\mathrm{kg} / \mathrm{m}^{3}\right)\end{array}$ & $\begin{array}{l}\text { Biomass } \\
\text { (tons) }\end{array}$ & $\begin{array}{l}\text { Carbon stock } \\
\text { (tons) }\end{array}$ & $\mathrm{CO}_{2}$ (tons) \\
\hline 95 & $"$ & Khaya ivorensis & 402 & 36410.11 & 530 & 19297.36 & 9648.68 & 35410.65 \\
\hline 96 & $"$ & Khaya senegalensis & 5587 & 134180.238 & 550 & 73799.13 & 36899.57 & 135421.41 \\
\hline 97 & $"$ & Lovoa trichilioides & 2040 & 211.32 & 582 & 122.99 & 61.49 & 225.68 \\
\hline 98 & $"$ & Milicia execlsa & 6269 & 263188.81 & 632 & 166335.33 & 83167.66 & 305225.33 \\
\hline 99 & $"$ & Trchilia emetica & 6 & 707.0154 & 608 & 429.87 & 214.93 & 788.80 \\
\hline 100 & $"$ & Trichilia lanata & 3 & 870.21 & 540 & 469.91 & 234.96 & 862.29 \\
\hline 101 & $"$ & Trichilia monadelpha & 7 & 824.8513 & 608 & 501.51 & 250.75 & 920.27 \\
\hline 102 & Mimosoideae & Acacia senegalensis & 9 & 597.5795 & 570 & 340.62 & 170.31 & 625.04 \\
\hline 103 & $"$ & Piptandeniastrum africanum & 1043 & 16968.8881 & 695 & 11793.38 & 5896.69 & 21640.85 \\
\hline 104 & Moraceae & Antiaris africana & 4788 & 312990.86 & 430 & 134586.07 & 67293.03 & 246965.44 \\
\hline 105 & $"$ & Artocarpus altillis & 247 & 28871.61 & 310 & 8950.20 & 4475.10 & 16423.62 \\
\hline 106 & $"$ & Astonia congensis & 634 & 288.7 & 460 & 132.80 & 66.40 & 243.69 \\
\hline 107 & $"$ & Ficus capensis & 21 & 717.0154 & 540 & 387.19 & 193.59 & 710.49 \\
\hline 108 & $"$ & Ficus exasperata & 10402 & 225599.643 & 600 & 135359.79 & 67679.89 & 248385.21 \\
\hline 109 & $"$ & Ficus thonningii & 2 & 235.6718 & 640 & 150.83 & 75.41 & 276.77 \\
\hline 110 & $"$ & Morus mesozygia & 4259 & 243155.163 & 230 & 55925.69 & 27962.84 & 102623.64 \\
\hline 111 & Myristicaceae & Coelocaryon preussii & 64 & 3656.7158 & 640 & 2340.30 & 1170.15 & 4294.45 \\
\hline 112 & Myrtaceae & Sizygium orwariense & 879 & 50222.706 & 460 & 23102.44 & 11551.22 & 42392.99 \\
\hline 113 & " & Syzgium guineense & 1 & 117.8359 & 665 & 78.36 & 39.18 & 143.79 \\
\hline 114 & Ochnaceae & Lophira alata & 1745 & 972.95 & 800 & 778.36 & 389.18 & 1428.29 \\
\hline 115 & Olacaceae & Strombosia graandifolia & 3 & 2.73 & 816 & 2.23 & 1.11 & 4.09 \\
\hline 116 & $"$ & Strombosia pustulata & 1041 & 21.1 & 804 & 16.96 & 8.48 & 31.13 \\
\hline 117 & Palmea & Borassus aethiopum & 1214 & 971.0772 & 640 & 621.49 & 310.74 & 1140.43 \\
\hline 118 & " & Bosqueia angolensis & 666 & 50750.099 & 600 & 30450.06 & 15225.03 & 55875.86 \\
\hline 119 & Papilionaceae & Baphia pubescens & 2 & 235.6718 & 640 & 150.83 & 75.41 & 276.77 \\
\hline 120 & $"$ & Berlinia confusa & 753 & 5033.2653 & 640 & 3221.29 & 1610.64 & 5911.07 \\
\hline 121 & $"$ & Berlinia congolensis & 1 & 117.8359 & 690 & 81.31 & 40.65 & 149.20 \\
\hline 122 & $"$ & Berlinia coriacea & 84 & 9898.215 & 690 & 6829.77 & 3414.88 & 12532.62 \\
\hline 123 & $"$ & Berlinia grandiflora & 192 & 22788.149 & 690 & 15723.82 & 7861.91 & 28853.21 \\
\hline 124 & $"$ & Lonchocarpus sericeus & 166 & 5990.633 & 540 & 3234.94 & 1617.47 & 5936.12 \\
\hline 125 & Papilionoideae & Amphimas pterocarpoides & 3384 & 72793.189 & 880 & 64058.01 & 32029.00 & 117546.44 \\
\hline 126 & Phyllanthaceae & Margaritaria discoidea & 6 & 707.0154 & 640 & 452.49 & 226.24 & 830.32 \\
\hline 127 & Rhabdodermatidae & Trilepisium madagacariense & 6 & 707.0154 & 518 & 366.23 & 183.12 & 672.04 \\
\hline 128 & Rhizophoraceae & Cassipourea barteri & 1 & 117.8359 & 640 & 75.41 & 37.71 & 138.39 \\
\hline 129 & $"$ & Cedrella odorata & 73 & 4114.7153 & 390 & 1604.74 & 802.37 & 2944.70 \\
\hline 130 & Rubiaceae & Anthocleista djalonensis & 19 & 2238.882 & 640 & 1432.88 & 716.44 & 2629.34 \\
\hline 131 & $"$ & Mitragyna ciliata & 4149 & 5865.5469 & 640 & 3753.95 & 1876.98 & 6888.50 \\
\hline 132 & $"$ & Mitragyna stipulosa & 8365 & 504.2477 & 470 & 237.00 & 118.50 & 434.89 \\
\hline 133 & $"$ & Nauclea diderrichii & 7587 & 1655.0536 & 780 & 1290.94 & 645.47 & 2368.88 \\
\hline 134 & $"$ & Pausinystalia johimbe & 2479 & 51.8 & 640 & 33.15 & 16.58 & 60.83 \\
\hline 135 & Rutaceae & Fagara rubescens & 17 & 363.0577 & 470 & 170.64 & 85.32 & 313.12 \\
\hline 136 & $"$ & Fagara santalinoides & 161 & 18971.58 & 640 & 12141.81 & 6070.91 & 22280.22 \\
\hline 137 & $"$ & Detarium microcarpum & 1352 & 57531.6429 & 690 & 39696.83 & 19848.42 & 72843.69 \\
\hline 138 & Sapindaceae & Blighia sapida & 1484 & 87985.671 & 750 & 65989.25 & 32994.63 & 121090.28 \\
\hline 139 & Sapotaceae & Aningeria robusta & 11 & 123.2759 & 640 & 78.90 & 39.45 & 144.78 \\
\hline 140 & " & Chrysophyllum albidum & 1979 & 95421 & 560 & 53435.76 & 26717.88 & 98054.62 \\
\hline 141 & $"$ & Chrysophyllum delevoyi & 1 & 3.02 & 560 & 1.69 & 0.85 & 3.10 \\
\hline
\end{tabular}


Table 2. Continued

\begin{tabular}{|c|c|c|c|c|c|c|c|c|}
\hline $\mathrm{S} / \mathrm{N}$ & Family & Tree Species & $\begin{array}{c}\text { Number } \\
\text { of tree }\end{array}$ & Volume $\left(\mathrm{m}^{3}\right)$ & $\begin{array}{l}\text { Density } \\
\left(\mathrm{kg} / \mathrm{m}^{3}\right)\end{array}$ & $\begin{array}{c}\text { Biomass } \\
\text { (tons) }\end{array}$ & $\begin{array}{c}\text { Carbon stock } \\
\text { (tons) }\end{array}$ & $\mathrm{CO}_{2}$ (tons) \\
\hline 142 & $"$ & Vitellaria paradoxa & 1 & 117.8359 & 640 & 75.41 & 37.71 & 138.39 \\
\hline 143 & Simaroubaceae & Hannoa ferruginea & 21 & 143.4359 & 540 & 77.46 & 38.73 & 142.13 \\
\hline 144 & $"$ & Hannoa klaineana & 1179 & 34715.359 & 640 & 22217.83 & 11108.91 & 40769.72 \\
\hline 145 & " & Hannoa undulata & 85 & 10016.05 & 540 & 5408.67 & 2704.33 & 9924.90 \\
\hline 146 & Sterculiaceae & Mansonia alttissima & 637 & 24578.6954 & 672 & 16516.88 & 8258.44 & 30308.48 \\
\hline 147 & " & Nesogordonia papaverifera & 1036 & 17580.876 & 784 & 13783.41 & 6891.70 & 25292.55 \\
\hline 148 & $"$ & Pterygota macrocarpa & 1837 & 34565.1266 & 592 & 20462.55 & 10231.28 & 37548.79 \\
\hline 149 & " & Sterculia oblonga & 1120 & 5017.0671 & 600 & 3010.24 & 1505.12 & 5523.79 \\
\hline 150 & " & Sterculia rhinoptela & 644 & 28112.92 & 640 & 17992.27 & 8996.13 & 33015.81 \\
\hline 151 & " & Sterculia tragacantha & 278 & 5901.0666 & 640 & 3776.68 & 1888.34 & 6930.21 \\
\hline 152 & $"$ & Triplochiton scleroxylon & 2195 & 104983.86 & 384 & 40313.80 & 20156.90 & 73975.83 \\
\hline 153 & Ulmaceae & Celtis adolfi friderrei & 5 & 589.1795 & 832 & 490.20 & 245.10 & 899.51 \\
\hline 154 & $"$ & Celtis zenkerii & 2983 & 150401.7287 & 832 & 125134.24 & 62567.12 & 229621.33 \\
\hline 155 & $"$ & Holoptelea grandis & 1085 & 16650.6 & 650 & 10822.89 & 5411.45 & 19860.00 \\
\hline 156 & Verbenaceae & Gmelina arborea & 102 & 4359.153 & 540 & 2353.94 & 1176.97 & 4319.48 \\
\hline 157 & $"$ & Tectona grandis & 15 & 1161.8 & 660 & 766.79 & 383.39 & 1407.06 \\
\hline 158 & $"$ & Vitex doniana & 11 & 1296.195 & 510 & 661.06 & 330.53 & 1213.04 \\
\hline 159 & $"$ & Vitex grandifolia & 41 & 4127.426 & 540 & 2228.81 & 1114.41 & 4089.87 \\
\hline 160 & $"$ & Vitex simplicifolia & 5465 & 309900.44 & 510 & 158049.22 & 79024.61 & 290020.33 \\
\hline 161 & & Other spp & 859 & 5678.09 & 640 & 3633.98 & 1816.99 & 6668.35 \\
\hline & Total & & 246786 & 8321595.64 & & 4560724.36 & 2280362.18 & 8368929.20 \\
\hline & Mean & & 82262 & 2773865.21 & & 1520241.45 & 760120.73 & 2789643.10 \\
\hline
\end{tabular}

$\mathrm{CO}_{2}$ (0.65 tons). In Osun State, Ceiba pentandra that was also the most exploited species had the highest amount of $\mathrm{CO}_{2}$ (145,185.92 tons). This was followed by Milicia excelsa $(135,174.97$ tons) and the least exploited species was Cola millenii also had the least amount of $\mathrm{CO}_{2}$ (70.06 tons). In Ekiti State, Antiaris africana (39,680.58 tons) and Dialium guineense (32,655.87 tons) has the highest carbon release while the least carbon release was recorded for Baphia spps (1.77 tons).

On the whole, the total number of timber removed from the selected states' forests stood at about 246,885 trees, belonging to 161 different species and 45 families. This amounted to an annual removal of about 82262 trees. The total volume of logs exploited in the study area for the three year period stood at 8.3 million $\mathrm{m}^{3}$ and the annual removal is 2.8 million $\mathrm{m}^{3}$. The results revealed that Ceiba pentandra was the most exploited tree species in the zone with annual cut of about 5,625 trees with an annual volume of $213,340.35 \mathrm{~m}^{3}$. This is followed by Ficus exasperata with an annual cut of 3,467 trees and a volume of $75,199.88 \mathrm{~m}^{3}$.
The total biomass estimate of the trees from the selected forests for the 3-year period when their respective density values were applied was $4.64 \mathrm{Gt}$, with an annual tree biomass removal of 1.52 metric tons.

After the total volume of wood was converted to biomass for carbon estimation, the quantum of carbon represented by the total removal for the 3 -year period was estimated and it stood at 2.3 million metric tons from the 246,885 trees of the different tropical indigenous hardwood species. The annual carbon release therefore stood at 760,120.73 metric tons from a yearly cut of 82,262 trees. The estimated carbon in the logged woods was subsequently used to obtain the quantity of $\mathrm{CO}_{2}$ that the logged woods have the capacity of absorbing from the atmosphere. This translated to a total of 8.5 million metric tons of $\mathrm{CO}_{2}$, for the 3 -year period, that the removed woods of this region have the ability to sequestrate if they were to remain live standing trees. If these woods were burnt, about this amount of $\mathrm{CO}_{2}$ would have been released into the atmosphere. The annual carbon and $\mathrm{CO}_{2}$ equivalents are 760,120.73 metric tons and 2,789,643.10 
Table 3. Summary of result for the number of stems, volume and $\mathrm{CO}_{2}$ released from the study area

\begin{tabular}{lccccccc}
\hline State & $\begin{array}{c}\text { Mean no of } \\
\text { stems }\end{array}$ & $\begin{array}{c}\text { No of } \\
\text { species families }\end{array}$ & $\begin{array}{c}\text { No of } \\
\text { Mean volume }\end{array}$ & Biomass & Carbon & ${\text { Mean } \mathrm{CO}_{2}}$ \\
\hline Oyo & $4756 \pm 300.47^{\mathrm{a}}$ & 58 & 29 & $271724.46 \pm 17167.82^{\mathrm{a}}$ & $1586730.20 \pm 12273.10^{\mathrm{a}}$ & $79336.51 \pm 6136.55^{\mathrm{a}}$ & $291164.00 \pm 22521.13^{\mathrm{a}}$ \\
Osun & $814 \pm 132.25^{\mathrm{b}}$ & 130 & 42 & $102057.75 \pm 16804.84^{\mathrm{b}}$ & $450915.86 \pm 4256.05^{\mathrm{b}}$ & $22545.79 \pm 2128.03^{\mathrm{b}}$ & $88121.99 \pm 8830.20^{\mathrm{b}}$ \\
Ondo & $4102 \pm 511.67^{\mathrm{a}}$ & 61 & 24 & $16017.69 \pm 2776.92^{\mathrm{c}}$ & $86873.29 \pm 1381.41^{\mathrm{c}}$ & $4343.67 \pm 690.71^{\mathrm{c}}$ & $15941.25 \pm 2534.89^{\mathrm{c}}$ \\
Ogun & $23 \pm 1.12^{\mathrm{c}}$ & 74 & 33 & $1427.42 \pm 189.24^{\mathrm{d}}$ & $7011.57 \pm 73.21^{\mathrm{d}}$ & $350.58 \pm 36.60^{\mathrm{d}}$ & $1286.62 \pm 134.33^{\mathrm{d}}$ \\
Ekiti & $808.27 \pm 80.11^{\mathrm{b}}$ & 60 & 25 & $7497.41 \pm 2566.27^{\mathrm{c}}$ & $44529.55 \pm 1615.83^{\mathrm{c}}$ & $2226.48 \pm 807.92^{\mathrm{c}}$ & $7848.25 \pm 134.33^{\mathrm{c}}$ \\
\hline
\end{tabular}

*Means in the same column with the same superscript are not significantly different $(p>0.05)$.

metric tons respectively. It should be noted however that these estimates were for timbers legally removed and recorded in the department's monthly report of timber felled in the States. They were those removed by timber contractors or concessionaires with all necessary permits obtained from the government. There were no records for all the logs that were illegally removed from the reserves and those felled from the free areas.

The results of the one way analysis of variance revealed a significant difference $(p<0.05)$ in the number of trees, number of species, number of families, volume, biomass, carbon and the amount of $\mathrm{CO}_{2}$ released from the logged trees in the states. However, the estimates of these variables were found to be significantly higher in Oyo State when compared with the other four states. According to the mean separation results, there was significant difference $(\mathrm{p}>$ 0.05 ) in all these variables as shown in Table 3. Also, the list of ten most exploited tree species in each State is presented in Table 4. According to the Table, Mitragyna stipulosa $(8,353)$ recorded the highest number of exploitation for the three years period in Ondo State. In Oyo State, Anogeissus leicocarpus (6,590), had the highest number of exploitation but Osun, Ogun and Ekiti State, Ceiba pentandra was most exploited as presented in the Table.

\section{Discussion}

Trees exploited from the forests of South-West Nigeria by loggers were majorly tropical hardwood species of economic and timber values. Nowadays, virtually all species of trees including the lesser known and those that were not regarded as durable ones in the past are now logged for sawn wood production. This was due to the fact that the most economic species are disappearing due to high demand. In Nigeria, logging is usually from the forest reserves and the free areas. There are a total of 1600 productive forest reserves in Nigeria but their numbers and sizes varied from one state to the other. It has been reported that some of them only exist today on paper (Oyebo 2008). Apart from the wanton and indiscriminate logging activities, which had led to the degradation and destruction of most of these reserves, there are also the problems of encroachment and conversion to farmland by landless farmers and replacement with plantations of fast growing exotic species by the large-scale afforestation projects of both the State and Federal government. Two of the reserves in the zone (Omo and Oluwa Forest Reserves in Ondo and Ogun States, respectively) have been converted to large-scale afforestation projects of the government. These were the largest and most diverse forest reserves in the tropical rainforest ecosystem of southwest Nigeria. The fact that there is the scarcity of the most desirable species for timber production in the country today has resulted into having Ceiba pentandra, a species with poor timber quality and durability, as the most exploited species in the zone. This species has found its way to the timber market and even became very popular today.

We discovered that Ogun, the state with the largest forestland area, produced the least amount of annual timber. This may be due to the fact that the largest single forest reserve in the state (Omo Forest reserve) has an active large afforestation project. This large forest reserve that ought to be a good source of indigenous timber now housed a very large hectare of plantations of Gmelina arborea and Nauclea diderrichii under the World Bank Afforestation project with counterpart funding from the Federal Government of 


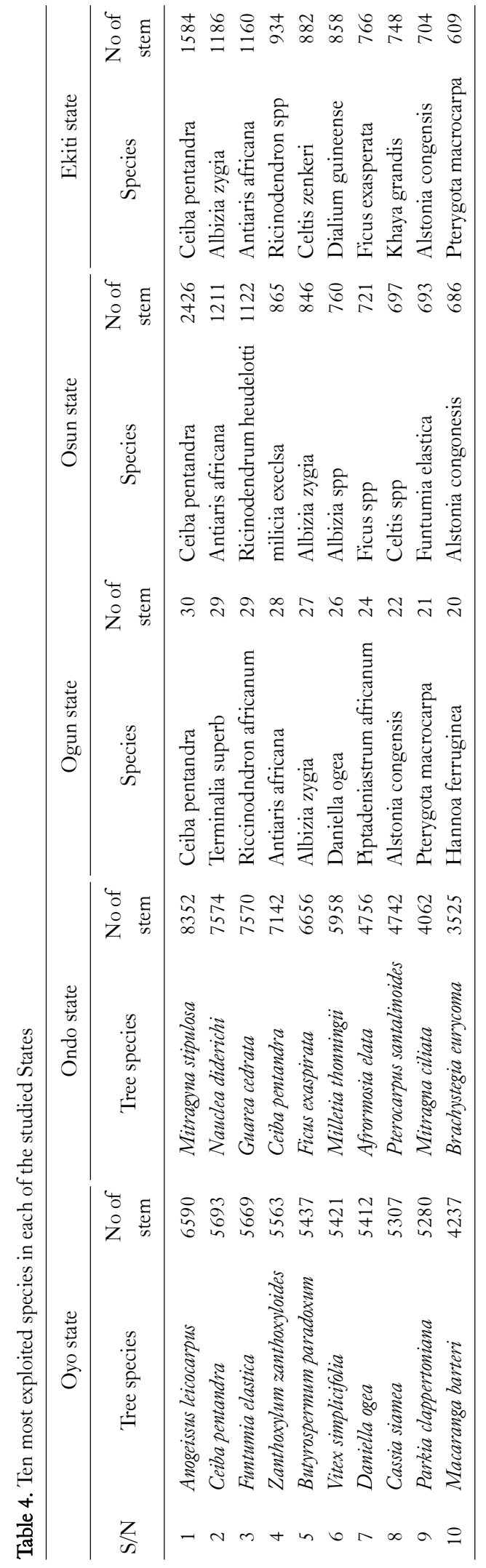

Nigeria and Ogun State Government. In addition, the relics of the reserve have been put under strict protection and constituted as Biosphere reserve by the Forestry Research Institute of Nigeria. The few cut recorded in the state was from the few productive reserves in Ogun State. Bulk of the state's log supply is from the neighbouring states.

In Nigeria, logging policy has to do with the allocation of compartments to concessionaires by the Forestry Department after the payment of all necessary levies. Logging in each compartment is through selective method. The involvement of the government in logging activities in the free areas (unreserved forests) is limited to issuance of log certificate after the production of owner's consent by the loggers. There are so many ecological and social problems with unsustainable logging in the reserves due to lack of former education and training for loggers and operators of felling machines, poor skidding methods, improper supervision by forest officers and corruption of some government forest staff. Some of the consequences of logging in the tropical rainforest ecosystem of south west Nigeria were reported by Adekunle and Ige (2006) and Adekunle and Olagoke (2010) to include loss of biodiversity, compaction of soil, destruction of the home of wildlife, opening of forest to encroachers and farmers and global warming.

The colossal annual volume of timber ( 8.2 million cubic meters) and the high number of trees $(231,000)$ that were removed from the forest ecosystem according to this study are detrimental to forest conservation for posterity, ecological and environmental benefits. With this present rate of logging in Nigeria, it seems that forest degradation is been disregarded. As a result, Nigerian forests are losing much of their carbon. This is very inimical to the implementation of the REDD agreement. There is also the loss of biodiversity and other ecosystem services, when valuable trees are harvested without regard to the ecological consequences (Broadbent et al. 2008; Schwenk et al. 2012). Meyfroidt and Lambin (2008) stated that political instability and governance failures, wildfires as well as the uncontrolled and often illegal logging usually result in forest degradation and deforestation, which has continued unabated in the tropics. Oyebo (2006) puts the annual deforestation rate in Nigeria at $3 \%$. Similarly, Bond et al. (2009) reported that logging and poor management is leading to the loss of approximately 400,000 hectares of tropical forest in the Congo 
Basin each year. Therefore, the problems of unsustainable logging are common scenario in most developing countries of West African sub-Sahara.

The contribution of big diameter logs in tropical rainforest ecosystem to carbon stock cannot be overestimated (e.g. Slik et al. 2013). The annual and mean monthly carbon estimates from wood biomass in this study are very enormous for some species with large number of big trees. Wood density was reported to be an important factor in converting forest volume data to biomass (Feamside 1997). Carbon content presented in this study was obtained from biomass estimated as the product of volume and density (Petsri et al. 2007). High quantity carbon release estimated could be related to the huge annual timber harvest from the study forests. Howbeit, the results of this work aligned with the assertion of Sasaki and Putz (2009) that the evergreen forest holds an average above-ground carbon stock of 121.2 $\mathrm{Mg} \mathrm{C} /$ ha of which $71.4 \mathrm{Mg} \mathrm{C}$ is in trees $\geq 45 \mathrm{~cm} \mathrm{DBH}$. It was also reported by Houghton (2003), Achard et al. (2004) and Gullison et al. (2007) that tropical deforestation and degradation globally will result in the release of an estimated 1.1-2.2 Pg Carbon/ year (It should be noted however that $1 \mathrm{Pg} \mathrm{C}=10^{15} \mathrm{~g} \mathrm{C}$ ). The amount of carbon obtained by Petstri et al. (2007) from live trees in a mixed deciduous forest was 71.6 metric tons/ha. But, Dahal (2008) reported that a single tree can absorb tons of $\mathrm{CO}_{2}$ over its lifetime, and protecting a young plant and forests is equally important as planting. Based on our result, we inferred that there is increasing pressure on economic tree species and it is plausible that they are becoming scarce from the forests in Southwestern Nigeria. Normally if such species economic trees are still available in abundance and within reach, we opine that lower number of commonly less-utilized species like Ceiba pentandra would have been exploited. The total annual carbon obtained in this study is an estimate from logs removed legally for sawn wood production only. Data were not available for those removed illegally.

Illegal logging according to Auer et. al. (2003) can be broadly defined as "large scale, destructive forest harvesting that transgresses the laws of the nation where said harvesting occurs. While Fuwape (2001), highlighted some causes of forest destruction to include illegal logging, accelerated urbanization, conversion of forest reserves to farmlands and housing estate, Contreras-Hermosilla (2003) itemize some specific activities of illegal logging to include the following: logging protected species; duplication of felling licenses; girdling or ring-barking, to kill trees so that they can be legally logged; contracting with local entrepreneurs to buy logs from protected areas; logging in protected areas; logging outside concession boundaries; logging in prohibited areas such as steep slopes, riverbanks, and water catchments; removing under/oversized trees from public forests; extracting more timber than authorized; reporting high volumes of timber extracted in forest concessions to mask the volume taken from areas outside concession boundaries and obtaining logging concessions through bribes. Consequently, each day at least 80,000 acres $(32,300 \mathrm{ha})$ of forest either disappear or degraded from the Earth (Imeht and Adebobola 2001). The planet loses as many as several hundred species to extinction, the vast majority of which have never been documented by science and it is not even certain that all the Earth biodiversity would be documented before they are extinct.

As the forests are degraded, more carbon is added to the atmosphere, climactic conditions are further altered, and more topsoil is lost to erosion. Abubaka (2010) also reported that forest destruction is usually accompanied by reduction in species diversity, decrease in primary productivity, change in forest structure and depletion in soil nutrients. Poverty, corruption and population increase are some of the factors responsible for massive cut in Nigeria. Therefore to avert those consequences that may arise as a result of massive forest destruction, government should ensure that log harvesting is sustainable. Sustainable log harvesting can be achieved through the determination of an Allowable Annual Cut (AAC) and minimum dbh. An Allowable Annual Cut (AAC) refers to a harvest level that is partially calculated and then further adjusted to reflect socio-economic values and issues within the management unit for which it has been determined. The purpose of determining an AAC is to ensure that harvest levels does not exceed growing levels and that harvest levels are sustainable.

The results of this research indicated that the highest amount of carbon and $\mathrm{CO}_{2}$ were in Oyo State where the highest number of logs were exploited. This could be attributed to the fact that the state has the highest population when compared with the other states. More wood and for- 
estland are required by the large number of people in the state. IPCC (2001) attributed the rise in $\mathrm{CO}_{2}$ concentration and emission to human activities such as burning of fossil fuel, land use change and deforestation. The anthropogenic activities such as slash and burn, fire management, timber harvesting (indiscriminate logging) and encroachment have accelerated the release of Green House Gases from the forests (Canadell et al. 2007). These activities are very rampant Nigerian forest estates and they resultantly have adverse effect on biodiversity conservation, ecosystem services and carbon storage. They are also very inimical to sustainable forest management. Furthermore, stand density was discovered to be a factor affecting the increase in carbon content of the location (Petsri et al. 2007).

The roles of forest ecosystem in the global carbon cycle cannot be over estimated. IPCC (2001) reported that forest stores about $80 \%$ of all above-ground and $40 \%$ of all below-ground terrestrial organic carbon. During productive season, it was claimed that $\mathrm{CO}_{2}$ from the atmosphere is taken up by vegetation (Losi et al. 2003; Phat et al. 2004) and stored as plant biomass. In view of this, the UNFCC and its Kyoto Protocol recognized the role of forests in carbon sequestration. Specifically, Article 3.3 and 3.4 of the Kyoto Protocol pointed out forest as potential carbon storage (Brown 2002). At the present rate of logging in the tropical forest ecosystem of south west Nigeria, coupled with all other sources of natural and human influences, much carbon is been released into the atmosphere than the amount needed by vegetation for photosynthesis. It has been reported that forest disturbances could lead to the release of carbon greater than what the plants will require for their regular activities (Brown 2002). Sustainable management strategies are therefore necessary to make the forest a carbon sink rather than source. Accounting for the carbon within forest ecosystems and changes in carbon stocks resulting from human activities is a necessary first step towards the better representation of forests in climate change policy at regional, national and global scales (Canadell et al. 2007). Hence, there is immediate need for the control of logging and forest degradation in the developing countries.

\section{Conclusion and Recommendation}

The role of forest ecosystem in carbon sequestration is critical to climate change adaptation and mitigation. The huge volume of wood removed annually from the Nigerian tropical rainforest ecosystem as demonstrated in this study and other human activities are precursory to deforestation and carbon emission. Based on our result, we inferred that there is increasing pressure on economic tree species and it is plausible that they are becoming scarce from the forests in Southwestern Nigeria. If the present rate of log removal is not controlled, forests could become carbon source rather than carbon sink and the on biological conservation, wood availability and climate change may turn out grave. For the forest to perform its environmental role as carbon sink, urgent conservation measures and logging policies are needed to be put in place in this country. Indiscriminate Logging without regeneration negates the Clean Development Mechanism policy. As a result, urgent conservation measure is recommended. Also, appropriate logging policies in the reserves and free areas, afforestation and reforestation and awareness of the effect of the continuous logging activities on the climate should be put in place by all tiers of government and individual in sub Saharan Africa.

\section{Acknowledgement}

We wish to thank the Directors of Forestry in the selected states for making logging data available for this work.

\section{References}

Aboala JR, Arévalob JR, Fernández Á. 2005. Allometric relationships of different tree species and stand above ground biomass in the Gomera laurel forest (Canary Islands). Flora - Morphology, Distribution, Functional Ecology of Plants 200: 264-274.

Abubaka HT. 2010. Many Species One Planet One Future. A Keynote Address. In: Ofoezie IE, Awotoye OO, Adewole MB (eds), Proceeding of the $3^{\text {rd }}$ Annual Conference of the Institute of Ecology and Environmental Studies, OAU, Ile Ife, Nigeria. June $15^{\text {th }}-17^{\text {th }}, 2010$.

Achard F, Eva HD, Mayaux P, Stibig HJ, Belward A. 2004. Improved estimates of net carbon emissions from land cover change in the tropics for the 1990s. Global Biogeochemical Cy 18: GB2008.

Adekunle VAJ, Ige PO. 2006. Logging and Logging Residues of some Selected Economic Tropical Hardwood Timber Species in Free Areas of Ondo State, Nigeria. Appl Trop Agr 11: 81-92. 
Adekunlea VAJ, Olagokea AO. 2010. The impacts of timber harvesting on residual trees and seedlings in a tropical rain forest ecosystem, southwestern Nigeria. Int J of Biod Sci, Ecosyst Serv Manag 6: 131-138.

Adesodun JK, Adeyemi EF, Oyegoke CO. 2007. Distribution of nutrient elements within water-stable aggregates of two tropical agro-ecological soils under different land uses. Soil Till Res 92: 190-197.

Auer MR, Ingram D, Farley C. 2003. Towards an Improved Understanding of Illegal Logging and Associated Trade, U.S. Forest Service, International Programs (Washington, DC, May 30, 2003).

Birdsey RA. 1996. Carbon storage for major forest types and regions in the coterminous United States. In: Forests and global change Vol. 2 Forest management opportunities for mitigating carbon emissions (Sampson RN, ed). American Forests, Washington, DC, pp 1-25.

Bond I, Grieg-Gran M, Wertz-Kanounnikoff S, Hazlewood P, Wunder S, Angelsen A. 2009. Incentives to sustain forest ecosystem services: A review and lessons for REDD. Natural Resource Issues No. 16. International Institute for Environment and Development, London, UK, with CIFOR, Bogor, Indonesia, and World Resources Institute, Washington D.C., USA, pp 62.

Broadbent EN, Asner GP, Keller M, Knapp DE, Oliveira PJC, Silvad JN. 2008. Forest fragmentation and edge effects from deforestation and selective logging in the Brazilian Amazon. Biol Cons 141: 1745-1757.

Brown S. 1997. Estimating biomass and biomass change of tropical forests: a Primer. FAO Forestry Paper 134.

Brown S. 2002. Measuring carbon in forests: current status and future challenges. Environ Pollut 116: 363-372.

Canadell JG, Le Quéré C, Raupach MR, Field CB, Buitenhuis ET, Ciais P, Conway TJ, Gillett NP, Houghton RA, Marland G. 2007. Contributions to accelerating atmospheric $\mathrm{CO}_{2}$ growth from economic activity, carbon intensity, and efficiency of natural sinks. Proc Natl Acad Sci U S A 104: 18866-18870.

Contreras-Hermosilla A. 2003. Forest Law Enforcement: An Overview, World Bank Discussion Paper (Washington, DC: June 19, 2003).

Dahal N. 2008. Answering Basic Questions on Climate Change in Nepal. NGO Group Bulletin on Climate Change Research and Development, Issue 2 August 2008, Published by the Local Initiatives for Biodiversity, research and Development (LIBIRD) in association with NGO group on Climate Change Nepal and PROLINNOVA Nepal Programme.

Dinwoodie JM. 1981. Timber its nature and behaviour. Van Nosttrand Reinhold Company Ltd., Molly Millars Lane, Wokingham, Berkshire, England, pp 190.

Drake JB, Knox RG, Dubayah RO, Clark DB, Condit R, Blair JB, Hofton M. 2003. Above-ground biomass estimation in closed canopy Neotropical forests using lidar remote sensing: factors affecting the generality of relationships. Global Ecol Biogeogr 12: $147-159$.

Fearnside PM. Wood density for estimating forest biomass in Brazilian Amazonia. Forest Ecol Manag 90: 59-87.

Fuwape JA. 2001. Forest resources and economic development in Ondo State Economic summit, $2-5^{\text {th }}$ April, 2001, pp 1-15.

Gullison RE, Frumhoff PC, Canadell JG, Field CB, Nepstad DC, Hayhoe K, Avissar R, Curran LM, Friedlingstein P, Jones CD, Nobre C. 2007. Tropical Forests and Climate Policy. Science 316: 985-986.

Hiratsuka M, Toma T, Yamada M, Heriansyah I, Morikawa Y. 2004. A general allometric equation for estimating biomass in Acacia mangium plantations. In: Proceedings of the 2003 International Conference on Tropical Forests and Climate Change : TFCC 2003 : carbon sequestration and clean development mechanism : 21-22 October 2003, Traders Hotel, Roxas Blvd., Manila, Philippines (University of the Philippines at Los Baños. College of Forestry and Natural Resources, eds). University of the Philippines Los Banos, College of Forestry and Natural Resources, Manila, Philippines, pp 212-218.

Hiratsuka M, Toma T, Diana R, Hadriyanto D, Morikawa Y. 2006. Biomass Recovery of Naturally Regenerated Vegetation after the 1998 Forest Fire in East Kalimantan, Indonesia. JARQ 40: 277-282.

Houghton RA. 2003. Revised estimates of the annual net flux of carbon to the atmosphere from changes in land use and land management 1850-2000. Tellus B 55: 378-390.

Huq S, Reid H, Murray L. 2003. Mainstreaming Adaptation to Climate Change in Least Developed Countries (LDCs) Working Paper 1: Country-by-Country Vulnerability to Climate Change, International Institute for Environment and Development, 42p London. http://www.iied.org/climate_change/pubs. html April 2003.

IISD 2009. A Summary of the Third International Workshop on Community-Based Adaptation to Climate Change Bulletin Online at HTTP:/WWW.IISD.CA/YMB/SDCAB/ Volume 135, No. 2, Friday, 27 Feb 2009 pp 13.

Imeht N, Adebobola N. 2001. The effects of poverty in conservation of Biodiversity: The Nigeria Experience. http://www. scienceinafrica.co.20

IPCC (Intergovernmental Panel on Climate Change) 2006. IPCC Guidelines for National Greenhouse Gas Inventories. Prepared by the National Greenhouse Gas Inventories Programme (Eggleston HS, Buendia L, Miwa K, Ngara T, Tanabe K, eds). Institute for Global Environmental Strategies, Japan.

IPCC (Intergovernmental Panel on Climate Change) 2001. Climate Change 2001: Working Group I: The Scientific Basis. Cambridge University Press, New York.

Kraenzel M, Castillo A, Moore T, Potvin C. 2003. Carbon storage of harvest-age teak (Tectona grandis) plantations, Panama. Forest Ecol Manag 173: 213-225.

Laclau P. 2003. Biomass and carbon sequestration of ponderosa 
pine plantations and native cypress forests in northwest Patagonia. For Ecol Manage 180: 317-333.

Losia CJ, Siccama TG, Condit R, Morales JE. 2003. Analysis of alternative methods for estimating carbon stock in young tropical plantations. For Ecol Manage 184: 355-368.

Macdicken KG. 1997. Project specific monitoring and verification: State of the art and challenges. Mitigat Adapt Strategies Global Change 2: 27-38.

Meyfroidt P, Lambin EF. 2008. Forest transition in Vietnam and its environmental impacts. Glob Change Biol 14:1-8.

Montagu KD, Düttmer K, Barton CVM, Cowie AL. 2005. Developing general allometric relationships for regional estimates of carbon sequestration - an example using Eucalyptus pilularis from seven contrasting sites. For Ecol Manage 204: 115-129.

Montès N, Gauquelin T, Badrib W, Bertaudière V, Zaouic EH. 2000. A non-destructive method for estimating above-ground forest biomass in threatened woodlands. For Ecol Manage 130: 37-46.

NIACS (Northern Institute of Applied Carbon Science) 2008. Estimating Carbon Mass in Northern Forests. NIACS Briefing (Janowiak MK - Briefing contributor), 410 MacInnes Drive, Houghton, MI 49931, pp 3.

NCP (Nigerian Standard Code of Practice) (1973). Nigerian Standard Code of Practice. Times press Apapa, Nigeria, pp 71.

NPC (National Population Commission) 1991. Report of the country's population census, Abuja, Nigeria.

Oyebo MA. 2006. History of forest management in Nigeria from 19th century to date. In: Imperatives of space technology for sustainable forest management in Nigeria: [proceedings of an international stakeholders' workshop sponsored by National Space Research and Development Agency (NARSDA), Abuja] (Salami AT, Geoformin, eds) Space Applications \& Environmental Science Laboratory, Obafemi Awolowo University, Ile-Ife, Nigeria, pp 1-14.

Penman J. 2003. Good Practice Guidance for Land Use, Land Use Change and Forestry. Intergovernmental Panel on Climate Change Working Group-National Greenhouse Gas Inventories Program.

Petsri S, Pumijumnong N, Wachrinrat C, Thoranisorn S. 2007. Aboveground carbon content in mixed deciduous forest and teak plantations. Environ Nat Res Journal 5: 1-10.

Phat NK, Knorr W, Kim S. 2004. Appropriate measures for conservation of terrestrial carbon stocks-Analysis of trends of for- est management in Southeast Asia. For Ecol Manage 191: 283-299.

Potter CS. 1999. Terrestrial biomass and the effects of deforestation on the global carbon cycle results from a model of primary production using satellite observations. Bioscience 49: 769-778.

Ranasingbe DM, Abayasiri S. 2008. Forestry Education and Global change: a case study on the contribution of forest plantations in Sri Lanka as an adaptation measure to climate. In: New perspectives in forestry education (Temu AB, African Network for Agroforestry Education, et al, eds). Peer review papers presented at the 1st Global Workshop on forestry education, Sept 2007. ANAFE, Nairobi, pp 353-360.

Samalca IK, Gier A, Hussin YA. 2007. Estimation of tropical forest biomass for assessment of carbon sequestration using regression models and remote sensing in Berau, East Kalimantan, Indonesia. Department of Natural Resources, The International Institute for Geoinformation Science and Earth Observation (ITC), Hengelosstraat 99, 7500 AA, Enschede, Netherlands, $6 \mathrm{p}$.

Sasaki N, Putz FE. 2009. Critical need for new definitions of "forest" and "forest degradation" in global climate change agreements. Wiley Periodicals, Inc. DOI: 10.1111/j.1755263X.2009.00067.x, Published online on 20 Jul 2009, pp15.

Schwenk WS, Donovan TM, Keeton WS, Nunery JS. 2012. Carbon storage, timber production, and biodiversity: comparing ecosystem services with multi-criteria decision analysis. Ecol Appl 22: 1612-1627.

Slik JWF, Paoli G, McGuire K, Amaral I, Barroso J, Bastian M, Blanc L, Bongers F, Boundja P, Clark C, Collins M, Dauby G, Ding Y, Doucet J-L, Eler E, Ferreira L, Forshed O, Fredriksson G, Gillet J-F, Harris D, Leal M, Laumonier Y, Malhi Y, Mansor A, Martin E, Miyamoto K, Araujo-Murakami A, Nagamasu H, Nilus R, Nurtjahya E, Oliveira Á, Onrizal O, Parada-Gutierrez A, Permana A, Poorter L, Poulsen J, Ramirez-Angulo H, Reitsma J, Rovero F, Rozak A, Sheil D, Silva-Espejo J, Silveira M, Spironelo W, ter Steege H, Stevart T, Navarro-Aguilar GE, Sunderland T, Suzuki E, Tang J, Theilade I, van der Heijden G, van Valkenburg J, Van Do T, Vilanova E, Vos V, Wich S, Wöll H, Yoneda T, Zang R, Zhang M-G, and Zweifel N. 2013. Large trees drive forest aboveground biomass variation in moist lowland forests across the tropics. Global Ecology and Biogeography 22: 1261-1271.

Wheeler T, von Braun J. 2013. Climate Change Impacts on Global Food Security. Science 341: 508-513. 